\title{
Experimental Study of the Intra-Channel Nonlinearity Influence on Single-Band 100G Coherent Optical OFDM Systems
}

\author{
Qunbi Zhuge, Xian Xu, Mohammad E. Mousa-Pasandi, Mohamed Morsy-Osman, Mathieu Chagnon,
} Ziad A. El-Sahn, and David V. Plant, Fellow, IEEE

\begin{abstract}
We report on an experimental investigation of the intra-channel nonlinearity effects in single-band 100G coherent optical orthogonal frequency division multiplexing (CO)-OFDM systems. We first show that instead of using traditional single-polarization training symbols (TS's), which suffer from nonlinearity-induced nonuniform phase shifts, correlated dual-polarization TS's should be used in reducedguard-interval CO-OFDM systems with short symbol durations. Next, we demonstrate that the intra-channel nonlinearity tolerance in CO-OFDM systems can be improved by employing fewer subcarriers for signal generation.
\end{abstract}

Index Terms-Channel estimation, fiber nonlinearity, orthogonal frequency-division multiplexing (OFDM).

\section{INTRODUCTION}

D UE TO the limited bandwidth of digital-to-analog converters (DACs), 100G coherent optical orthogonal frequency-division multiplexing (CO-OFDM) systems were previously realized using multiband (or superchannel) techniques [1], [2]. Moreover, the influence of fiber nonlinearities has been experimentally investigated in such systems [3], [4]. Owing to the availability of high speed DACs, recently singleband $112 \mathrm{~Gb} / \mathrm{s}$ (28 Gbaud QPSK) CO-OFDM transmission, which simplifies the hardware complexities as it only needs a single transmitter and receiver, has been demonstrated [5].

In this letter, we conduct an experimental investigation of the effects of intra-channel fiber nonlinearity in $100 \mathrm{G}$ singleband CO-OFDM systems. We first experimentally verify that the design of training symbols (TS's) impacts reducedguard-interval (RGI) [6] and conventional CO-OFDM systems differently when the intra-channel nonlinearity is nonnegligible [7]. Next, by varying the inverse fast Fourier transform (IFFT) size from 32 to 1024 in RGI CO-OFDM systems, we quantitatively show that the intra-channel nonlinearity tolerance is reduced as the number of OFDM subcarriers increases.

Manuscript received November 1, 2012; revised December 27, 2012; accepted January 22, 2013. Date of publication January 30, 2013; date of current version February 22, 2013.

The authors are with the Department of Electrical and Computer Engineering, McGill University, Montreal, QC H3A 2A7 Canada (e-mail: qunbi.zhuge@mail.mcgill.ca; xian.xu@mail.mcgill.ca; me.mousapasandi@ mail.mcgill.ca; mohamed.osman2@mail.mcgill.ca; mathieu.chagnon@mail. mcgill.ca; ziad.elsahn@ieee.org; david.plant@mcgill.ca).

Color versions of one or more of the figures in this letter are available online at http://ieeexplore.ieee.org.

Digital Object Identifier 10.1109/LPT.2013.2243722

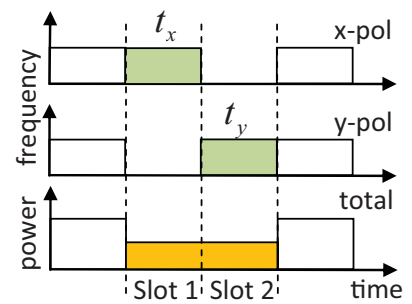

(a)

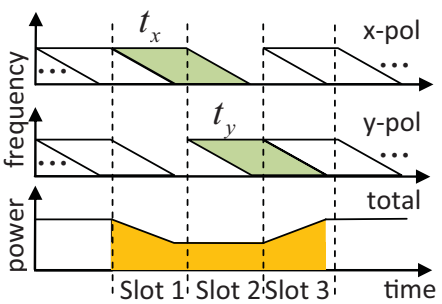

(b)
Fig. 1. Power profile of SP-TS's (a) before transmission and (b) during transmission in RGI CO-OFDM systems with a short symbol duration.

\section{IMPACT OF CHANNEL ESTIMATION}

RGI CO-OFDM systems compensate chromatic dispersion (CD) before OFDM demodulation, and therefore offer the flexibility to use a short OFDM symbol (with a small IFFT size) in order to increase the system tolerance to laser phase noise, frequency offset and fiber nonlinearities [6], [7]. However, when the symbol duration is short relative to the CDinduced walk-off between subcarriers, the traditional singlepolarization (SP) TS's [2] are not suited for RGI systems as has already shown in our previous simulation work [7]. This is because the power profile of the TS's becomes nonuniform as the signal propagates over the fiber, leading to nonuniform nonlinear phase shifts for different subcarriers in the TS's as illustrated in Fig. 1. The phase shifts can be described as,

$$
\varphi_{x(y)}=-\gamma\left(P_{x(y)}+K P_{y(x)}\right) L_{E f f}
$$

where $\gamma$ is the fiber nonlinearity coefficient, $K$ is the crosspolarization phase modulation coupling factor, and $L_{e f f}$ is the effective length. Specifically in Fig. 1(b) where the training symbols are spread to time slot 3 by the CD-induced walk-off, as less signal power is present in time slot 2 , less nonlinearity phase shift is applied to the subcarriers in time slot 2 than those in time slot 1 and 3, As a consequence, the channel transfer function estimated from the SP-TS's contains undesired phase shifts, which will be applied to data subcarriers through channel equalization, resulting in a performance degradation.

The correlated dual-polarization (CDP)-TS's, $t_{1}=\left[t_{x}, t_{y}\right]^{T}$, $t_{2}=\left[t_{x},-t_{y}\right]^{T}$, were first proposed to mitigate cross phase modulation (XPM) in wavelength-division multiplexing (WDM) CO-OFDM systems [8]. In this letter, we show that CDP-TS's can also increase the intra-channel nonlinearity tolerance due to the different phenomenon as described above. Particularly, because there is no power difference between 


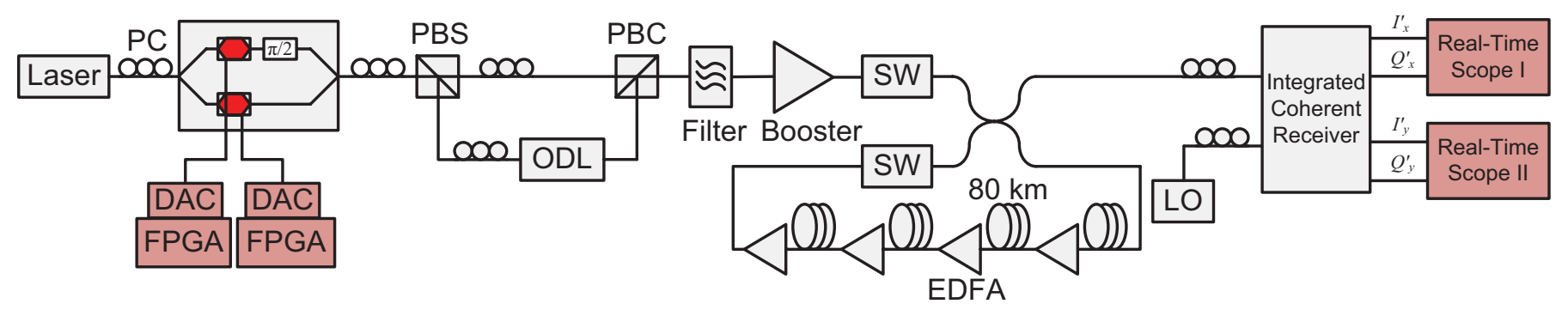

Fig. 2. Experimental setup (ODL: optical delay line. PBS/PBC: polarization beam splitter/combiner. PC: polarization controller. SW: switch.).

TS's and data symbols, the CDP-TS's don't experience intrachannel nonlinearity induced nonuniform phase shifts. The channel transfer function can be calculated by

$$
\begin{aligned}
& {\left[\begin{array}{l}
a(k) b(k) \\
c(k) d(k)
\end{array}\right]} \\
& \quad=\frac{1}{2}\left[\begin{array}{l}
{\left[t_{1 x}^{\prime}(k)+t_{2 x}^{\prime}(k)\right] / t_{x}(k)\left[t_{1 x}^{\prime}(k)-t_{2 x}^{\prime}(k)\right] / t_{y}(k)} \\
{\left[t_{1 y}^{\prime}(k)+t_{2 y}^{\prime}(k)\right] / t_{x}(k)\left[t_{1 y}^{\prime}(k)-t_{2 y}^{\prime}(k)\right] / t_{y}(k)}
\end{array}\right]
\end{aligned}
$$

where $\left[t_{1 x}^{\prime}, t_{1 y}^{\prime}\right]^{T},\left[t_{2 x}^{\prime}, t_{2 y}^{\prime}\right]^{T}$ are the received TS's [8].

\section{EXPERIMENTAL SETUP AND RESUlts}

Fig. 2 plots the experimental setup. Two field-programmable gate array (FPGA) boards were used to store the OFDM time domain samples and drive two $32 \mathrm{GS} / \mathrm{s}$ Micram DACs with 6 bit resolution to generate the analog inphase and quadrature signals. After electrical-to-optical conversion, the optical OFDM signal was passed through a dual-polarization (DP) emulator with a proper delay to align the TS's. The formed DP CO-OFDM signal was filtered to remove the image caused by the small oversampling ratio $(=1.14 \%)$, and boosted before transmission. The optical re-circulating loop consisted of 4 spans of $80 \mathrm{~km}$ standard single mode fiber (SMF-28e+) and 4 erbium doped fiber amplifiers (EDFAs) with $5 \mathrm{~dB}$ noise figure. The output signal was detected using an integrated and colorless coherent receiver. Four transimpedance amplifiers integrated in the receiver were used to amplify the electrical signals before digitization. External-cavity lasers (ECL's) were employed. The offline processing included re-sampling, synchronization, frequency offset compensation, channel estimation and equalization, and phase estimation. For RGI systems, a frequency domain $\mathrm{CD}$ equalizer was used before the OFDM demodulation. A pair of TS's (either SP-TS's or CDP-TS's) with the same length as data symbols was sent for the channel estimation, where intra-symbol frequency averaging (ISFA) with a filter length of 5 symbols was used to refine the estimate. For all systems, 4 pilot subcarriers were employed for coarse phase estimation, followed by a maximum-likelihood (ML) algorithm for fine phase estimation.

First, we investigated the impact of the intra-channel nonlinearity on the performance of SP-TS's and CDP-TS's for both RGI and conventional CO-OFDM systems. The IFFT size was 128 (112 filled subcarriers) and 2048 (1792 filled subcarriers), and the cyclic prefix (CP) length was 4 samples $(3.13 \%)$ and 460 samples (22.4\%) for the RGI and conventional CO-OFDM in this experiment, respectively. The raw data rate is $112 \mathrm{~Gb} / \mathrm{s}$ for all systems and we mainly investigate the performance at

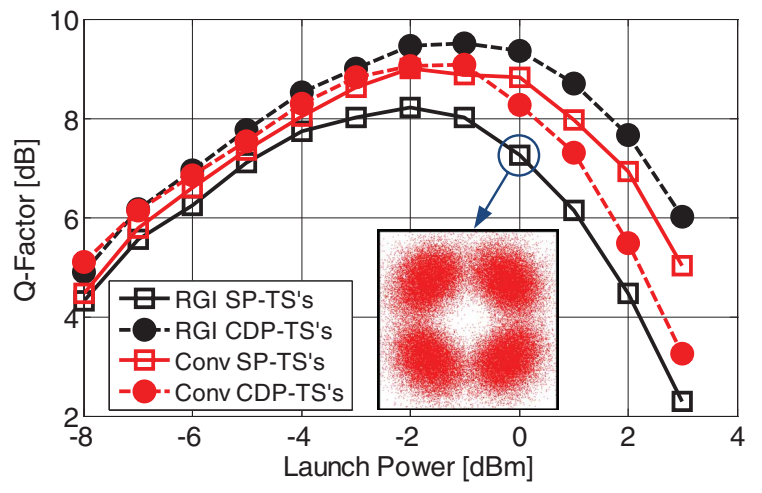

Fig. 3. Q-factor versus launch power for the RGI and conventional (Conv) CO-OFDM systems with different types of TS's. Inset: the constellation that shows the nonuniform phase shifts induced by the fiber nonlinearity.

the distance with a bit error rate (BER) close to $3.8 \times 10^{-3}$ (7\% hard-decision forward error coding (FEC) limit).

Fig. 3 shows the Q-factor calculated from BER versus the launch power at $3200 \mathrm{~km}$. For low powers $(<-6 \mathrm{dBm})$, all systems perform similarly with CDP-TS's performing slightly better than SP-TS's. This can be explained by the inherent averaging over two symbols for CDP-TS's in Eq. (2). As the power increases to the nonlinearity-limited regime, the performance starts to differ. Since the CD-induced walkoff is relatively shorter with respect to the symbol duration, the conventional CO-OFDM with SP-TS's doesn't experience severe nonuniform phase shifts as described in Section II. Moreover, since SP-TS's contain less power than CDP-TS's, they induce less intra-channel nonlinearity, leading to a higher Q-factor when the power is larger than $0 \mathrm{dBm}$ in Fig. 3 . However, for the RGI CO-OFDM, the SP-TS's suffer from nonuniform phase shifts, which dominate the performance degradation as shown in the constellation (inset). Therefore, in spite of the smaller nonlinearity experienced by SP-TS's, we observe a $1.3 \mathrm{~dB}$ lower optimal Q-factor for the system with SP-TS's than that with CDP-TS's. When comparing the performance of RGI and conventional CO-OFDM with CDP-TS's, we observe a $0.4 \mathrm{~dB}$ optimal Q-factor improvement for the RGI system, and the improvement is increasing as the power increases, e.g. $2.2 \mathrm{~dB}$ at $2 \mathrm{dBm}$.

As demonstrated by simulation in [9], the IFFT size $\left(N_{I F F T}\right)$, which is proportional to the number of subcarriers for a fixed oversampling ratio, affects the nonlinearity tolerance of OFDM systems. Unlike conventional CO-OFDM, $N_{I F F T}$ in RGI CO-OFDM is not restricted by the CD memory length (or the $\mathrm{CP}$ overhead). Therefore, it is necessary to quantitatively study the impact of $N_{I F F T}$ on the nonlinearity 


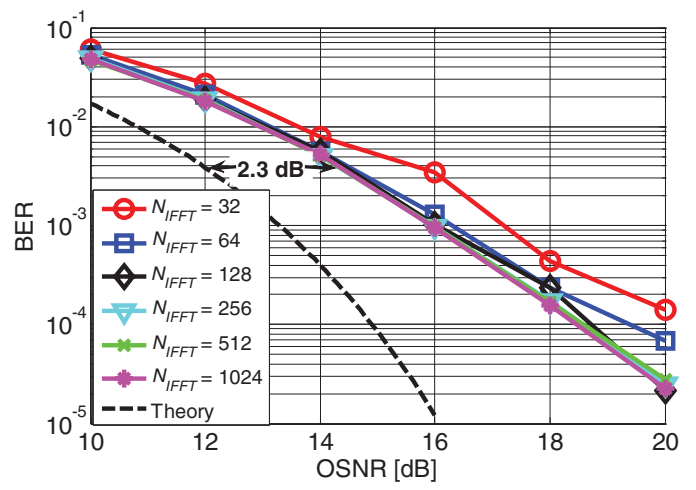

Fig. 4. BER versus OSNR for RGI CO-OFDM systems with various IFFT sizes.

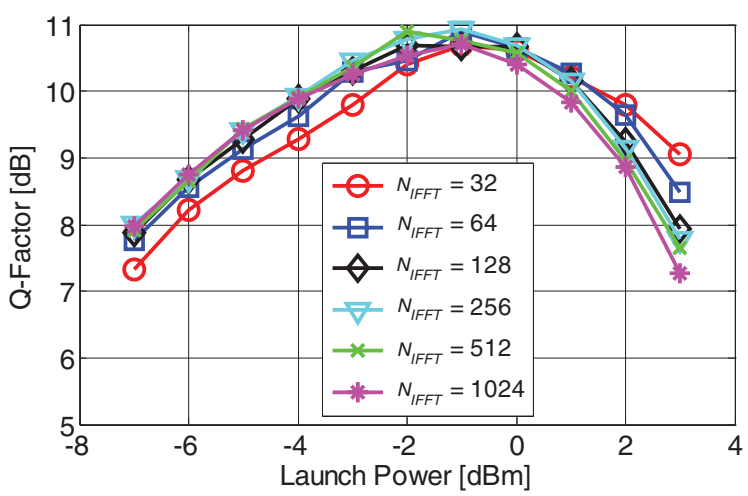

Fig. 5. Q-factor versus launch power for RGI CO-OFDM systems with various IFFT sizes at a $2240-\mathrm{km}$ distance.

tolerance in order to optimize the performance of RGI CO-OFDM systems. To do so, we conducted extensive measurements for the RGI systems (CDP-TS's) with various $N_{I F F T}$ ranging from 32 to 1024 .

First, we show the back-to-back performance of these systems in Fig. 4. A penalty is observed for the system with $N_{I F F T}=32$ compared to other systems, which might be caused by the reduced tolerance to the narrow filtering effect (there is an optical filter at the transmitter) and sampling frequency offset as the number of subcarriers decreases. In addition, with fewer subcarriers the efficiency of both the ISFA in channel estimation and ML phase estimation will be reduced.

Fig. 5 plots the Q-factor versus the launch power at $2240 \mathrm{~km}$. As reflected in the nonlinearity-limited regime, the nonlinearity tolerance increases as $N_{I F F T}$ is reduced. In particular, at $3 \mathrm{dBm}$ launch power, the system with $N_{I F F T}=32$ achieves a $0.6,1.1,1.3,1.4$ and $1.8 \mathrm{~dB}$ larger Q-factor than the system with $N_{I F F T}=64,128,256,512$ and 1024, respectively. However, the optimal Q-factors for all $N_{I F F T}$ values are very similar $(<0.25 \mathrm{~dB})$ and are partially limited by the effective number of bit of the DACs $(<5$ for frequencies $>8 \mathrm{GHz}$ ).

When the distance is increased to $4800 \mathrm{~km}$, the difference in the nonlinearity tolerance becomes more distinct for launched powers greater than $0 \mathrm{dBm}$ as shown in Fig. 6. For instance, at $3 \mathrm{dBm}$, a $0.5,1.4,1.7,2.3$ and $2.7 \mathrm{~dB}$ Q-factor improvement is observed for the system with $N_{I F F T}=32$ compared to the system with $N_{I F F T}=64,128,256,512$ and 1024 , respectively. In addition, the optimal launch power of

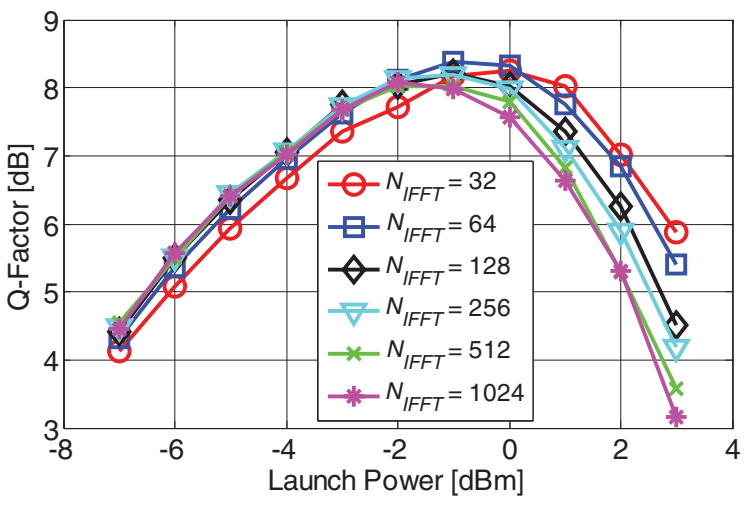

Fig. 6. Q-factor versus launch power for RGI CO-OFDM systems with various IFFT sizes at a $4800-\mathrm{km}$ distance.

$N_{I F F T}=32(0 \mathrm{dBm})$ is $2 \mathrm{~dB}$ larger than that of $N_{I F F T}=$ $1024(-2 \mathrm{dBm})$.

\section{CONCLusion}

In this letter, the influence of intra-channel nonlinearity is investigated in single-band $100 \mathrm{G}$ CO-OFDM systems. It is first shown that traditional single-polarization (SP) training symbols (TS's) experience undesired nonuniform phase shifts induced by nonlinearity in reduced-guard-interval (RGI) CO-OFDM systems. Then we experimentally demonstrated that the intra-channel nonlinearity tolerance of CO-OFDM is improved when the inverse fast Fourier transform (IFFT) size for signal generation is reduced.

\section{ACKNOWLEDGMENT}

The authors would like to thank TeraXion for providing the integrated Si-Photonic coherent receiver.

\section{REFERENCES}

[1] W. Shieh, Q. Yang, and Y. Ma, " $107 \mathrm{~Gb} / \mathrm{s}$ coherent optical OFDM transmission over 1000-km SSMF fiber using orthogonal band multiplexing," Opt. Exp., vol. 16, no. 9, pp. 6378-6386, 2008.

[2] S. L. Jansen, I. Morita, T. C. W. Schenk, and H. Tanaka, "121.9-Gb/s PDM-OFDM Transmission With 2-b/s/Hz Spectral Efficiency Over 1000 km of SSMF," J. Lightw. Technol., vol. 27, no. 3, pp. 177-188, Feb. 1, 2009.

[3] Y. Tang, Y. Ma, and W. Shieh, "Performance Impact of Inline Chromatic Dispersion Compensation for 107-Gb/s Coherent Optical OFDM," IEEE Photon. Technol. Lett., vol. 21, no. 15, pp. 1042-1044, Aug. 1, 2009.

[4] X. Chen, A. Li, G. Gao, and W. Shieh, "Experimental demonstration of improved fiber nonlinearity tolerance for unique-word DFT-spread OFDM systems," Opt. Exp., vol. 19, no. 27, pp. 26198-26207, 2011.

[5] Q. Zhuge, et.al., "Demonstration of Dispersion-Enhanced Phase Noise in RGI CO-OFDM Systems," IEEE Photon. Technol. Lett., vol. 24, no. 16, pp. 1446-1449, Aug. 15, 2012.

[6] X. Liu, S. Chandrasekhar, Z. Benyuan, P. J. Winzer, A. H. Gnauck, and D. W. Peckham, " $448-\mathrm{Gb} / \mathrm{s}$ reduced-guard-interval CO-OFDM transmission over $2000 \mathrm{~km}$ of ultra-large-area fiber and five 80-GHz-grid ROADMs," J. Lightw. Technol., vol. 29, no. 4, pp. 483-490, Feb. 15, 2011.

[7] Q. Zhuge, C. Chen, and D. V. Plant, "Impact of intra-channel fiber nonlinearity on reduced-guard-interval CO-OFDM transmission," in Proc. Opt. Fiber Commun. Conf. Exposit. Nat. Fiber Opt. Eng. Conf., Mar. 2011, pp. 1-3.

[8] X. Liu, F. Buchali, and R. W. Tkach, "Improving the nonlinear tolerance of polarization-division-multiplexed CO-OFDM in long-haul fiber transmission," J. Lightw. Technol., vol. 27, no. 16, pp. 3632-3640, Aug. 15, 2009.

[9] T. Kobayashi, et.al., "Over $100 \mathrm{~Gb} / \mathrm{s}$ Electro-Optically Multiplexed OFDM for High-Capacity Optical Transport Network," J. Lightw. Technol., vol. 27, no. 16, pp. 3714-3720, Aug. 15, 2009. 